\title{
Adsorption and Desorption Characteristic of Anesthetic Xenon on Silver Exchanged Zeolites
}

V. Sheth, A. Ritter- Biomedical Engineering, Chemistry and Biological Sciences, Stevens Institute of Technology, Hoboken, New Jersey, USA

\begin{abstract}
The purification/recycling of different components such as Xenon and Oxygen from anesthesia flow circuit's exhaust port, is an important economic feasible process for use of xenon for multiple patient use. Pressure Swing Adsorption (PSA) is surpassing the traditional stripping for many air separation applications, because of its lower energy consumption. Unfortunately, the Xenon product purity in a medical PSA process is typically limited to $80 \%$ due to the presence of oxygen which always shows the same adsorption equilibrium properties as nitrogen on most molecular sieves. Recent work investigating the adsorption of Xenon and oxygen on the surface of silver-exchanged Engelhard Titanosilicate-10 (ETS-10), indicates that this molecular sieve is promising as an adsorbent capable of producing high purity xenon on regeneration thereafter. The analysis has been evaluated by Aspen simulation software (Version 7.3) for Adsorption process. In this work Xenon was generated using a bed of Ag-ETS-10 granules to separate air (70\% Xe and $30 \% \mathrm{O}_{2}$ ) at $25^{\circ} \mathrm{C}$ and 2 psi with Xenon's recovery rate greater than $75 \%$.
\end{abstract}

Introduction: The separation of different gases from exhaust port of anesthesia flow loop such as xenon and oxygen is an utmost important step for economic evaluation for use of xenon for multiple use in medical application. Being scarce and expensive, a closed rebreathing system offers the optimum delivery method. Reducing waste through on-line recycling after regeneration will help xenon to find its place among anesthetic substances. Anesthetic xenon can be recovered and reused from patient to patient to make it economically competitive with current gold standard methods for inhaled anesthetics.

An air separation pressure swing adsorption (PSA) process uses zeolites as adsorbents, as they preferentially adsorb xenon to oxygen. The recovery of xenon depends strongly on the adsorbent selected. Silver exchanged Zeolites have been used commercially as adsorbents for Xenon-Oxygen separation [III]. A study has been investigated for utilization of silver exchanged zeolites such as Ag-ETS-10 as adsorbents in PSA processes [I]. In this case, the separation of Xenon from $\mathrm{O}_{2}$ is done by equilibrium method where the kinetic selectivity of oxygen over xenon has been investigated and employed for stripping xenon from oxygen. ETS-10, a titanosilicate molecular sieve, was found to favor xenon based on the sieving properties of the adsorbent and the difference in size between $\mathrm{O}_{2}$ molecules and xenon atoms. A two-step experimental study was reported here and this process tends to produce low pressure product and will require re-compression adding cost and complexity to the process.

In 2008, Kuznicki's group demonstrated that silver exchanged ETS-10, a mixed coordination titanosilicate molecular sieve, has an adsorptive selectivity for xenon over oxygen [III]. The selectivity, reached 1.0 in Henry's region, is the highest selectivity reported to date for a silver-exchanged sieve.

This paper describes the adsorptive separation performance of Ag-ETS-10 for pre-mixed gas mixture (70\% Xenon and $30 \%$ Oxygen) at ambient condition on Ag-ETS-10 using a laboratoryscale demonstration unit. An adapted chromatographic method was developed to improve the quantification of $\mathrm{O}_{2}$ and xenon in the product stream. The separation factor, breakthrough and desorption profiles are reported.

Sample Preparation: University of Alberta, Canada

* ETS-10 adsorbent was ion exchanged with silver nitrate in deionized water.

* The mixture is heated at $80^{\circ} \mathrm{C}$ for $1 \mathrm{hr}$ and silver exchanged ETS- 10 was dried at $100^{\circ} \mathrm{C}$ in air

* Ag-ETS-10 was pelletized at $69,000 \mathrm{KPa}$ for $3 \mathrm{~min}$ and sieved to obtain particle size $(0.85-1.68 \mathrm{~mm})$

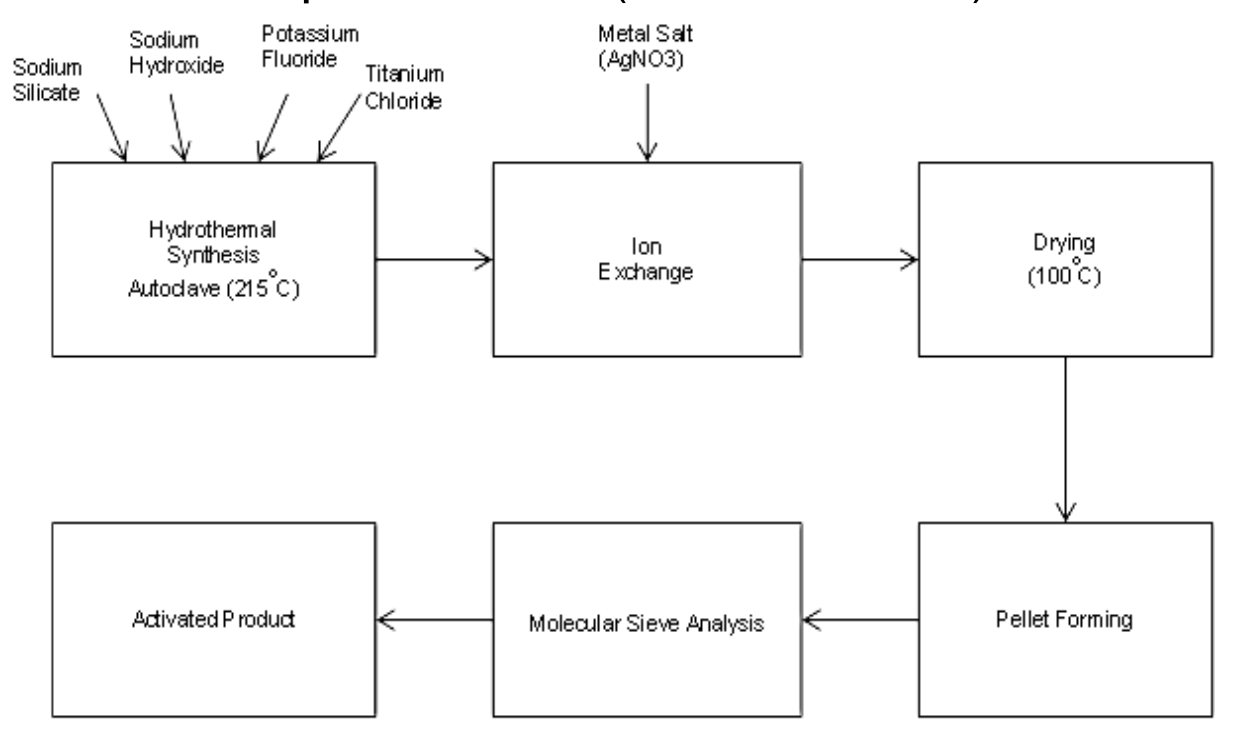

Laboratory Scale Set-up:

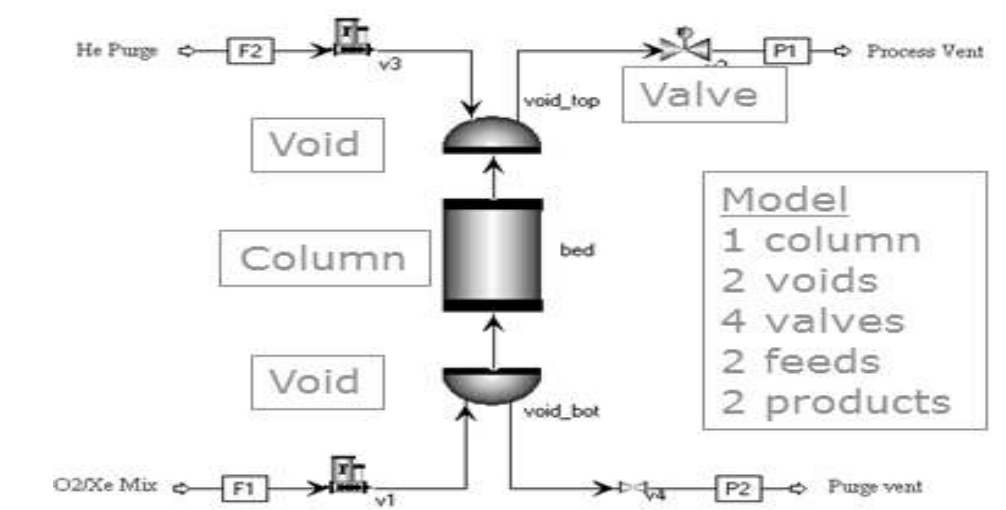

Schematic one stage process Xenon-Oxygen Adsorption on Ag-ETS-10

* $150 \mathrm{~g}$ adsorbent sample packed in $150 \mathrm{~cm}^{3}$ cylindrical stainless steel chamber with dia. of $5.03 \mathrm{~cm}$ and length of $13.33 \mathrm{~cm}$.

* The column packed with adsorbent pellets were activated at $200^{\circ} \mathrm{C}$ for $10 \mathrm{hr}$ under $100 \mathrm{~cm}^{3} / \mathrm{min}$ of helium flow

* Dry compressed air mixture of $\mathrm{Xe}$ and $\mathrm{O}_{2}$ was introduced in the fixed bed column at a flow rate of $60 \mathrm{~cm}^{3} / \mathrm{min}$ at $25^{\circ} \mathrm{C}$.

* Outlet gas composition analyzed using Gas Chromatography

Results and Discussion:

Adsorption Mechanism: Analysis of the separation of Xenon and Oxygen Ag-ETS- 10 at $25^{\circ} \mathrm{C}$. The retention time for xenon is larger than oxygen, indicating a preferred interaction between xenon and Ag-ETS-10[IV]
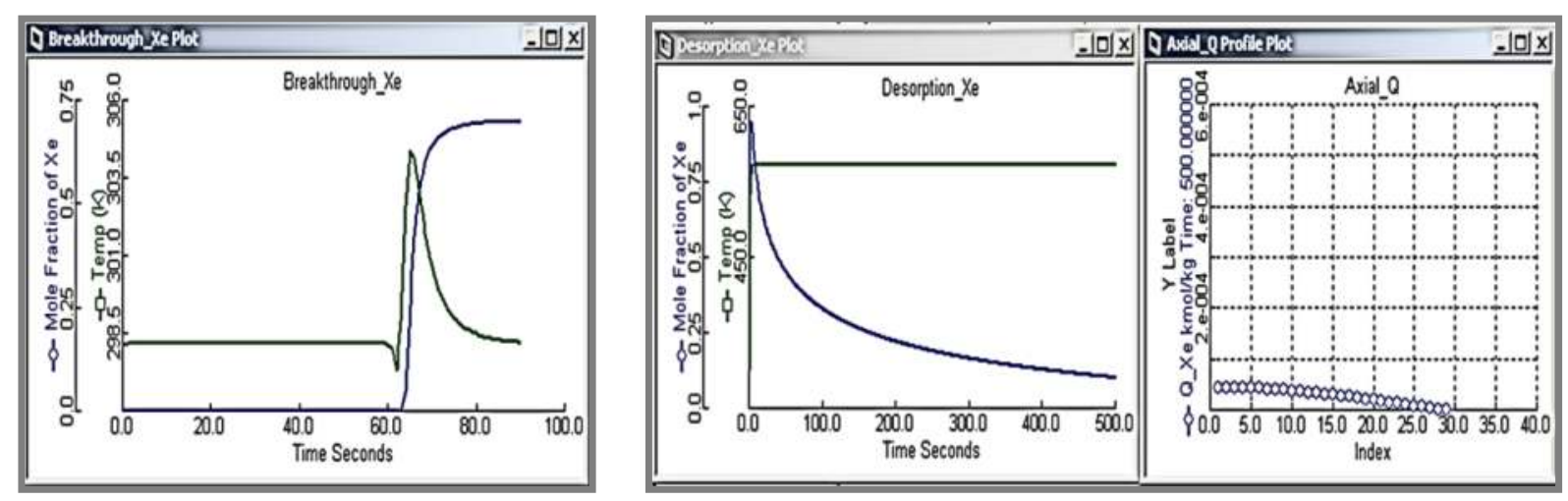

Desorption Mechanism: He purge $\mathrm{F}=2000 \mathrm{~mL} / \mathrm{min}$, purge $\mathrm{T}=$ $300^{\circ} \mathrm{C}$. Consumes lots of energy and purge gas for regeneration

\section{Conclusion:}

* Xenon was recovered at high yield and at a high concentration from a gas mixture containing $\mathrm{O}_{2}$ and $\mathrm{Xe}$

* The adsorbent exhibits quite high adsorption affinity for $\mathrm{Xe}$ at low pressure condition

- Theoretical value of the isosteric heat of adsorption $(83 \mathrm{~kJ} / \mathrm{mol})$ suggests this requires extensive energy for reactivation or regeneration of xenon

* This low-cost xenon anesthetic gas will be attractive for two broad applications: (1) Xenon will be the anesthetic agent of choice for large fraction of millions of surgical procedures that are performed across the globe on patients with cardiovascular conditions (2) because of fewer complications with rapid induction and emergence, xenon anesthesia can reduce patient time in hospitals, with large benefits to healthcare costs.

\section{References:}

I. Hill, T.L. "Stat. Mechanisms of Adsorption. V. Thermodynamics and Heat of Adsorption", J. Chem. Phys., 17, 520 (1949)

II. J. Yun et al, "Adsorption of Organic Solvent Vapors on Hydrophobic Y-Type Zeolite", AIChE J., 44, 1344 (1998)

III. S. M. Kuznicki et al, "Xenon Adsorption of Modified ETS-10", J. Phys. Chem. C, 111, 1560 (2007)

IV. Aspen Adsorption - Aspen Technology, Version 7.3. 